\title{
Evidence of injury caused by gas bubbles in a live marine mammal: barotrauma in a California sea lion Zalophus californianus
}

\author{
W. Van Bonn ${ }^{1, *}$, E. Montie ${ }^{2}$, S. Dennison ${ }^{1}$, N. Pussini ${ }^{1}$, P. Cook $^{3}$, D. Greig ${ }^{1}$, \\ J. Barakos ${ }^{4}$, K. Colegrove ${ }^{5}$, F. Gulland ${ }^{1}$ \\ ${ }^{1}$ Veterinary Science Department, The Marine Mammal Center, Sausalito, California 94965, USA \\ ${ }^{2}$ University of South Carolina Beaufort, Bluffton, South Carolina 29909, USA \\ ${ }^{3}$ Psychology Department, University of California at Santa Cruz, Santa Cruz, California 95064, USA \\ ${ }^{4}$ California Pacific Medical Center, University of California at San Francisco, San Francisco, California 94143, USA \\ ${ }^{5}$ Zoological Pathology Program, College of Veterinary Medicine, University of Illinois at Urbana-Champaign, Maywood, \\ Illinois 60153, USA
}

\begin{abstract}
A yearling male California sea lion Zalophus californianus with hypermetric ataxia and bilateral negative menace reflexes was brought to The Marine Mammal Center, Sausalito, California, USA, in late 2009 for medical assessment and treatment. The clinical signs were due to multiple gas bubbles within the cerebellum. These lesions were intraparenchymal, multifocal to coalescing, spherical to ovoid, and varied from 0.5 to $2.4 \mathrm{~cm}$ diameter. The gas composed $21.3 \%$ of the total cerebellum volume. Three rib fractures were also noted during diagnostic evaluation and were presumed to be associated with the gas bubbles in the brain. The progression of clinical signs and lesion appearance were monitored with magnetic resonance imaging, cognitive function testing and computed tomography. Gas filled voids in the cerebellum were filled with fluid on follow up images. Clinical signs resolved and the sea lion was released with a satellite tag attached. Post release the animal travelled approximately $75 \mathrm{~km}$ north and $80 \mathrm{~km}$ south of the release site and the tag recorded dives of over $150 \mathrm{~m}$ depth. The animal re-stranded $25 \mathrm{~d}$ following release and died of a subacute bronchopneumonia and pleuritis. This is the first instance of clinical injury due to gas bubble formation described in a living pinniped and the first sea lion with quantifiable cerebellar damage to take part in spatial learning and memory testing.
\end{abstract}

KEY WORDS: Gas bubbles · Barotrauma $\cdot$ California sea lion · Zalophus californianus

Resale or republication not permitted without written consent of the publisher

\section{INTRODUCTION}

Marine mammals possess exquisite biochemical, anatomical, physiological, and behavioural adaptations to life in the oceans, including mechanisms to prevent in vivo gas bubble formation during repetitive diving (Ridgway 1972, Elsner 1999). Elevated arterial blood nitrogen tensions have been documented in free-diving pinnipeds (Falke et al. 1985), and elevated muscle nitrogen tensions have been measured in trained dolphins following a series of dives (Ridgway \& Howard 1979). To date there have been no reported clinical cases of gas bubble formation in living marine mammals, although lesions in dead cetaceans associated with gas bubbles indicate bubbles have formed in vivo (Fernandez et al. 2005, Jepson et al. 2005). Houser and colleagues have recently re-visited the question of nitrogen saturation in the bottlenose dolphin Tursiops truncatus and concluded that nitrogen supersaturation is likely. However, they were unable to demonstrate vascular bubble formation in a repetitively diving trained animal (Houser et al. 2010).

In spite of the evidence for tolerance of elevated gas tensions, gas bubbles are found and sometimes associ- 
ated with injury in marine mammals. There are reports of gas bubbles discovered in various tissues during the necropsies of stranded marine mammals, some of which stranded in temporal and spatial association with mid-frequency active sonar, as well as in animals by-caught in nets (Jepson et al. 2003, 2005, Fernandez et al. 2005, Cox et al. 2006, Moore et al. 2009). Tissue reactions, including disseminated haemorrhages in lipid-rich tissues (Fernandez et al. 2005) and fibrous encapsulation of gas cavities (Jepson et al. 2005), indicate gas bubbles can occur in live animals and persist for long enough to generate cellular responses. In contrast to bubble formation as a result of supersaturation conditions, gas escaping from a normally gas-filled structure during change in ambient pressure with resultant physical damage is best classified as barotrauma. The true prevalence of injuries due to supersaturation and gas bubble formation or barotrauma in marine mammals is unknown (Gulland \& Hall 2007), and like gas bubble formation following supersaturation, naturally occurring barotrauma has never been reported in a living marine mammal.

Here we present the first clinical evidence of gas bubble injury in a living marine mammal. Gas bubbles were detected in the brain of a yearling sea lion that was brought to our rehabilitation hospital facility. Changes in the appearance of the lesions, the clinical signs and laboratory assessment of the animal were followed for over $100 \mathrm{~d}$. By combining magnetic resonance imaging studies with cognitive testing, this study also provides some insight into brain function in these unique animals.

\section{MATERIALS AND METHODS}

In September 2009, a $26.5 \mathrm{~kg}$ body weight, yearling male California sea lion Zalophus californianus was brought to The Marine Mammal Center (TMMC) for clinical care following rescue off a public dock where it was described as 'head weaving and lethargic'. On admission, clinical signs of hypermetric ataxia and bilateral negative menace reflexes were observed, suggesting a localized cerebellar lesion. During the entire stay at TMMC the animal was housed in a purpose-built rehabilitation enclosure with other conspecifics of the same age class and fed thawed, previously fresh-frozen herring at approximately $10 \%$ of body weight daily.

An initial health assessment was conducted upon arrival following standard TMMC protocol. Briefly, the animal was manually restrained, a standard body length measurement obtained, gender confirmed and the animal was thoroughly visually inspected, auscultated and palpated. Whole blood was collected by veinipuncture of the caudal gluteal vein using $\mathrm{K}^{+}$EDTA and serum separator Vacutainer ${ }^{\circledR}$ tubes (BD) and submitted to our laboratory for automated cell counts (Vet ABC Hematology Analyzer, Scil Animal Care Company), manual white cell differential determination and a serum chemistry panel (ACE, Alfa Wassermann).

Survey radiographs of the head and thorax were obtained under general anaesthesia $2 \mathrm{~d}$ after initial assessment. Anaesthesia was induced and maintained with isoflurane (Webster Veterinary) in oxygen delivered by mask using a semi-closed circle configuration. Respiration was spontaneous throughout. Orthogonal radiographic projections of the head and thorax were obtained using a fixed diagnostic x-ray unit and standard medical grade screen-film combinations. A cerebrospinal fluid (CSF) sample was collected while the animal was anaesthetized via dural puncture at the cisterna magna and submitted to an outside reference laboratory (IDEXX Laboratories) for fluid analysis. The animal was transported to a veterinary magnetic resonance imaging (MRI) facility (Animal Scan) $2 \mathrm{~d}$ after survey radigraphs were obtained. Anaesthesia was induced with sevoflurane (Webster Veterinary) in oxygen by mask. The animal was intubated with a cuffed endotracheal tube and maintained on isoflurane in oxygen using a semi-closed circle configuration. Respiration was mechanically controlled throughout the imaging period delivering a tidal volume of $250 \mathrm{ml}$ at a rate of 10 breaths $\mathrm{min}^{-1}$ and peak inspiratory pressure of $18 \mathrm{~cm}$ $\mathrm{H}_{2} \mathrm{O}$.

MRI scanning of the brain was completed with a 1.5$\mathrm{T}$ Siemens Magnetom Symphony scanner (Siemens) equipped with a CP Extremity Coil. Following the localizer scan, 2-dimensional proton density (PD) and T2-weighted (T2W) images in the transverse plane were acquired using a turbo spin-echo (TSE) sequence with the following parameters: time to repetition $(\mathrm{TR})=$ $3650 \mathrm{~ms}$; time to echo $(\mathrm{TE})=14 / 98 \mathrm{~ms}$ for $\mathrm{PD}$ and $\mathrm{T} 2 \mathrm{~W}$ respectively; slice thickness $=2.5 \mathrm{~mm}$; field of view $($ FOV $)=150 \times 150 \mathrm{~mm}$; voxel size $=0.3 \times 0.3 \times 2.5 \mathrm{~mm}$. Anatomical structures were identified using the MRIbased, brain atlas of the California sea lion (Montie et al. 2009). Initial evaluation of MR images was completed at the MRI unit. Post-processing, segmentation (i.e. assigning pixels to particular structures), 3D reconstructions, and volume analysis were performed using the software program AMIRA 4.1.1 (Mercury Computer Systems). Segmenting brain structures, creating 3-dimensional reconstructions, and volume analysis used methods described in the MRI-based brain atlas of a live California sea lion (Montie et al. 2009).

Recovery was uneventful and the animal returned to TMMC. Follow up radiographs and blood samples were obtained on Day 25 of hospitalization using methods described previously. 
The animal participated in cognitive testing during the rehabilitation period. Two tests were conducted, a motor-learning assay involving alternation in a $\mathrm{T}$ maze, and a long-term spatial memory assay involving regularly baited locations. The T-maze has been used in motor learning and spatial memory tests involving mice with cerebellum damage. The long-term spatial task described here is similar in structure to the Morris water maze, also frequently used in spatial learning and memory tasks involving mice with cerebellum damage (Petrosini et al. 1996).

The T-maze in this study consisted of a $\sim 3$ chute, open on one end and terminating at the other end in hinged doors, one on the right and one on the left. During experimental sessions, the animal was free to exit the chute from either door. The doors opened only outward, so having exited, the animal had to return to the entrance of the chute to re-enter the maze. During experimental sessions the animal was allowed to continuously navigate the T-maze. Each trip through the maze and selection of a door constituted a trial. On each trial, a fish reward was provided immediately following selection of the correct door: no reward was provided if the animal selected the incorrect door. The animal was rewarded for selecting either door on the first trial of an experimental session, but on following trials within the session, the correct door was the opposite of that selected on the trial immediately preceding. Thus, maximum reinforcement could be obtained by navigating the maze in an alternating pattern, left, right, left, right, etc. A variable number of trials were conducted on each day: no fewer than 20 and no more than 100. The animal continued participating in Tmaze sessions until meeting a criterion of $85 \%$ or more correct trials on 2 consecutive sessions of 20 trials each. Successful alternation during continuous navigation of a T-maze is believed to be a measure of procedural or motor learning (Wood et al. 2000).

In the long-term spatial memory task, 4 potential food locations - opaque buckets - were made available once every $24 \mathrm{~h}$. The same location was baited with fish on each presentation. For each daily presentation the buckets were made available until the animal had eaten all of the fish from the baited bucket and visited each of the other buckets at least once, or until the animal had eaten the fish and at least $5 \mathrm{~min}$ had passed since the buckets were presented. A 'visit' was defined as the animal's nose being within 6 in (ca. $15 \mathrm{~cm}$ ) of a bucket rim while head orientation was within 15 degrees of the bucket's midline. The buckets were presented on 12 separate occasions. This task was constructed to be similar to the Morris water maze task (Morris 1984), in which a mouse or rat must find a hidden, submerged platform and is then tested on their ability to re-locate the platform following delay. Initial learning of such tasks can be supported by procedural and motor memory, associative memory (e.g. the platform is next to the bolt), and allocentric spatial memory.

Following the $21 \mathrm{~d}$ period of cognitive testing, whole body computed tomography (CT) imaging was performed. The animal was transported to a 64-row multislice scanner (Lightspeed VCT $\circledR_{1}$, GE Healthcare). Anaesthesia was induced with isoflurane by mask. The animal was intubated with a cuffed endotracheal tube and maintained on isoflurane in oxygen delivered via semi-closed circle configuration. Respiration was spontaneous throughout. Proprietary bone and detail reconstruction algorithms (medium and high frequency reconstruction algorithms) with $1.25 \mathrm{~mm}$ slice thickness and pitch of 1.4 (single slice scanner definition) and gantry rotation time of $0.8 \mathrm{~s}$ were applied to acquire images of the entire body without contrast administration. Contrast administration into the subclavian vein for a post contrast series was attempted but was unsuccessful. Recovery from anaesthesia was uneventful and the animal again returned to TMMC enclosures.

A second MRI was obtained $73 \mathrm{~d}$ after initial presentation using the scanner and image acquisition protocol described previously. Immediately following imaging and while still under general anaesthesia, the animal was fitted with a satellite tag (SPLASH, Wildlife Computers) glued to the hair of the head with Loctite ${ }^{\text {тм }}$ 422 (Henkel). The tag was programmed to transmit binned dive data in $6 \mathrm{~h}$ intervals: 09:00-15:00, 15:0021:00, 21:00-03:00, and 03:00-09:00 h. Dive depth was sampled every $10 \mathrm{~s}$ and recorded in depth bins: 2-10, 10-20, 20-30, 30-40, 40-50, 50-60, 60-70, 70-80, 80$100,100-120,120-140,140-160$, and $>160 \mathrm{~m}$. Dive duration was recorded in time bins: $0,1,2,3,4,5,6,7$, $8,9,10,15,20,25$, and $>25$ s. Time at depth was recorded in the same depth bins as dive depth. The tag measured depth to the resolution of $0.5 \mathrm{~m}$ and dives less than $2 \mathrm{~m}$ were ignored.

On Day 74 whole blood was collected as described previously and complete blood counts, white cell differential determination and serum chemistries repeated. The animal was released from Chimney Rock, Point Reyes National Seashore (37.9902 ${ }^{\circ}$, $122.9639^{\circ} \mathrm{W}$ ) the following day.

The sea lion was found stranded again $25 \mathrm{~d}$ following release (99 d from initial presentation) inside San Francisco Bay $\left(36.805^{\circ} \mathrm{N}, 121.792^{\circ} \mathrm{W}\right)$. It was returned to TMMC and another health assessment conducted including complete blood count (CBC), white cell differential and serum chemistries. The animal was free from clinical signs of neurologic impairment and ate well. It was found dead in its enclosure $3 \mathrm{~d}$ later. The body was held overnight at refrigeration temperatures and a third MRI study of the head obtained the next 
day using the same scanner and image acquisition protocols described previously. Immediately following the MRI a complete necropsy examination was performed in accordance with standard TMMC protocols. Representative tissue sections from all organs were fixed in $10 \%$ neutral buffered formalin and submitted to a veterinary pathologist (Zoological Pathology Program) for examination and interpretation. Tissues were processed routinely, embedded in paraffin, and $5 \mu \mathrm{m} \mathrm{sec-}$ tions were stained with haematoxilin and eosin (H\&E). Select sections of brain were stained with antibodies to the endothelial marker Factor VIII (rabbit polycolonal, Dako). Sections of lung were stained with Brown and Hopps.

Swabs (Transporter ${ }^{\mathrm{TM}}$ HealthLink) of exudates recovered from the thoracic cavity for microbiological isolation and identification attempts were submitted to the California Animal Health and Food Safety Laboratory
System (California Department of Food \& Agriculture, Animal Health Branch).

\section{RESULTS AND DISCUSSION}

Results of the initial $\mathrm{CBC}$, serum chemistry panel and the CSF analyses were clinically unremarkable. For brevity, specifics of parameters and values for these and the repeated cell counts and chemistry analyses (also clinically unremarkable) are not shown.

The survey radiographs of the head revealed gas opacities throughout the caudal fossa. Radiographs of the thorax revealed non-displaced fractures of the left third, fourth and fifth ribs. The rib fractures showed slight rounding of fracture margins without evidence of callus formation suggestive of fractures between 1 and
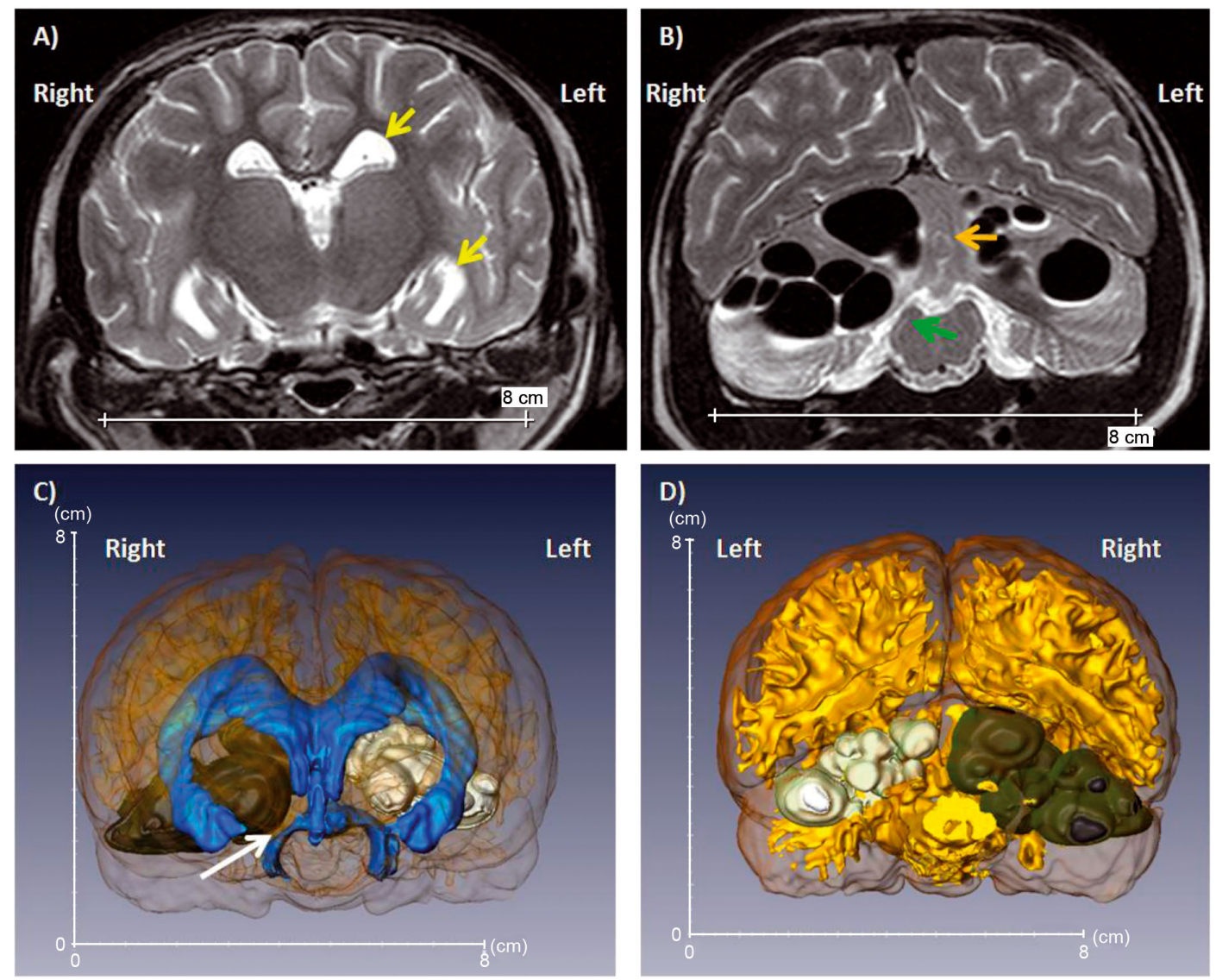

Fig. 1. Zalophus californianus. Initial magnetic resonance imaging (MRI). (A,B) T2-weighted images of the brain showing hydrocephalus and signal voids consistent with gas bubbles. These images were acquired in the transverse plane. The central and ventral horns of the lateral ventricle are enlarged (yellow arrows). Gas bubbles are visible in the cerebellum but not cerebral hemispheres. (C,D) 3-dimensional reconstructions of hydrocephalus and gas bubbles. In (C), the brain is viewed rostral to caudal, where grey and white matter is translucent, ventricles are blue, left air is white, and right air is dark green/brown. In (D), the brain is viewed caudal to rostral grey matter is translucent and ventricles are removed. The gas bubbles in the right cerebellum are larger in volume than the bubbles in the left cerebellum (15.25 compared to $\left.6.87 \mathrm{~cm}^{3}\right)$, causing a mass-effect, deforming and displacing the vermis (orange arrow in B) and right caudal cerebellar peduncle (green arrow in B), and shifting the mesencephalic aqueduct and fourth ventricle leftward (white arrow in C). The gas composed $21.3 \%$ of the cerebellum. Scale bars $=8 \mathrm{~cm}$ 
2 wk chronicity. There was no evidence of pneumonia or pneumothorax.

The first MRI of the brain revealed signal voids on T2W and PD images consistent with gas that were restricted to the cerebellum (Fig. 1). These lesions were intraparenchymal, multifocal to coalescing, spherical to ovoid, and varied from 0.5 to $2.4 \mathrm{~cm}$ diameter. The gas composed $21.3 \%$ of the total cerebellum volume. The volume of gas in the right cerebellum $\left(15.2 \mathrm{~cm}^{3}\right)$ was larger than the volume in the left cerebellum $\left(6.9 \mathrm{~cm}^{3}\right)$. This difference exerted a mass effect, which deflected the cerebellar vermis leftward and caused an outflow obstruction, resulting in mild hydrocephalus as compared to the normal sea lion brain (Montie et al. 2009). The presence and distribution of the signal void lesions correlated with the gas opacities observed in the radiographs within the caudal fossa, and a diagnosis of pneumocerebellum was made.
The CT performed 2 mo after the initial MRI revealed round to ovoid structures within the cerebellum, in similar locations and of similar size to the gas lesions identified using MRI. However, these lesions had Hounsfield units similar to CSF, indicating that the gas had resolved and was replaced by fluid. The calvarium, nasal cavity, and tympanic bullae were all normal ruling out head trauma as the cause of the gasleakage into the caudal fossa. Bony proliferation consistent with callus formation was identified in the proximal portion of the third, fourth and fifth left ribs, indicating that the rib fractures were healing and healing was consistent with the speculated time of trauma. There was no evidence of pneumonia or pneumothorax. The second MRI performed 2 wk after the CT demonstrated an alteration in the size and signal intensity of the cerebellar lesions (Fig. 2). The signal intensity from all lesions had changed from signal voids to
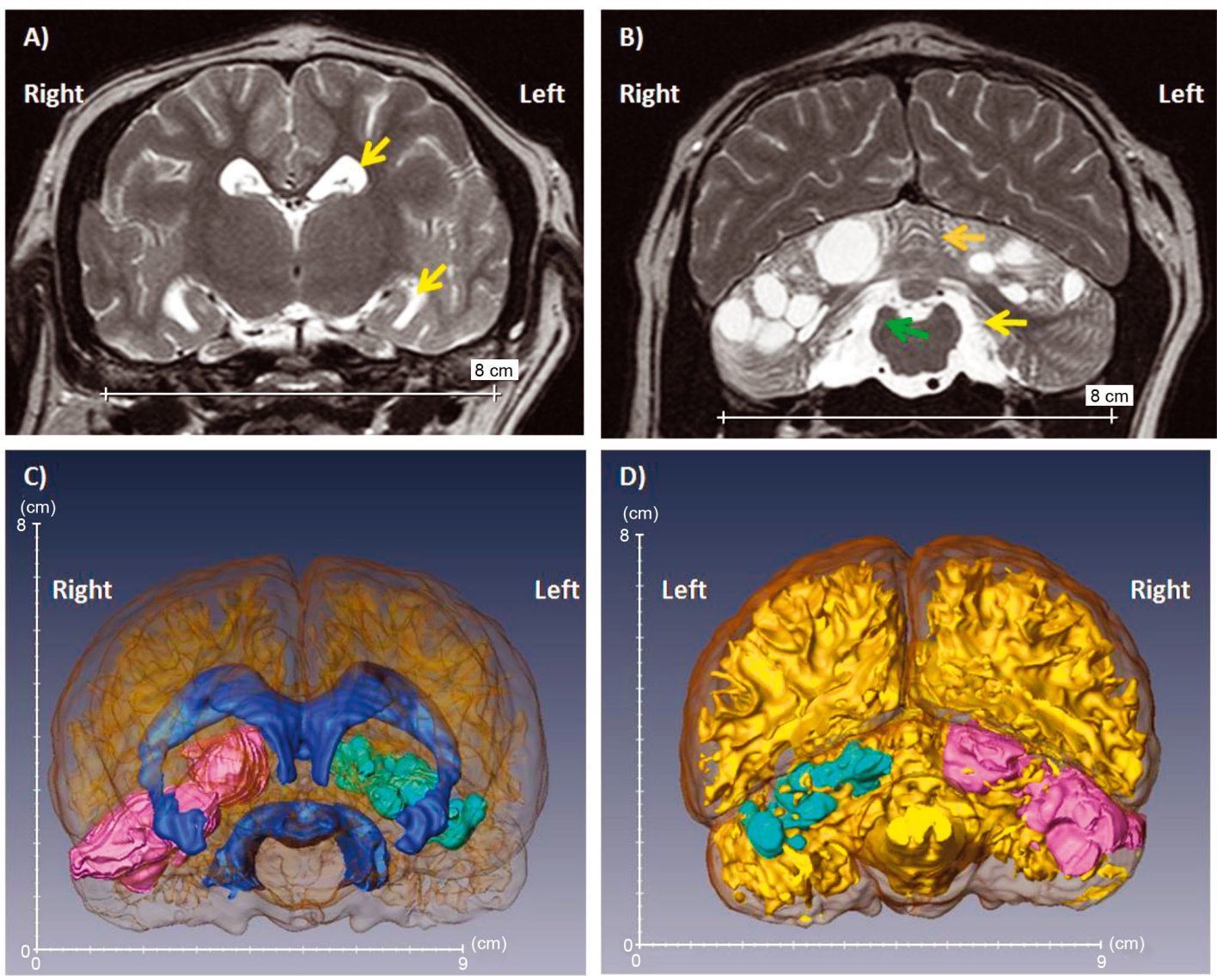

Fig. 2. Zalophus californianus. Follow up magnetic resonance imaging (MRI) showing fluid replacement of previously gas-filled vesicles. (A) Hydrocephalus is still present (yellow arrows), although it is less pronounced in the central and ventral horns of the lateral ventricles compared to the initial MRI. (B) Gas is now replaced with fluid. Hydrocephalus is more prominent in the lateral recess of the fourth ventricle as compared to the initial MRI (yellow arrow). (C,D) 3-dimensional reconstructions of hydrocephalus and fluid. In (C), the brain is viewed rostral to caudal, where grey and white matter are translucent, ventricles are blue, left fluid is green, and right fluid is pink. In (D), the brain is viewed caudal to rostral; grey mattter is translucent and ventricles are

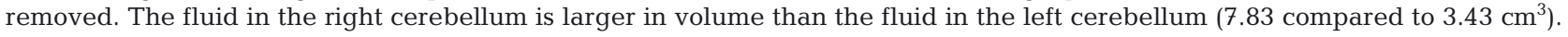
However, the mass-effect is not as predominant, and the vermis (orange arrow in B) and right caudal cerebellar peduncle (green arrow in B) are less deformed and displaced. The fluid composed $11.6 \%$ of the cerebellum. Scale bars in $(\mathrm{A})$ and $(\mathrm{B})=8 \mathrm{~cm}$ 
intensities similar to CSF on both T2W and PD sequences, consistent with CT findings of fluid-filled intraparenchymal cavitations. These findings indicated a progression of the cerebellar lesions to cystic encephalomalacia. The fluid in the right cerebellum remained larger in volume than the fluid in the left cerebellum ( 7.8 compared to $\left.3.4 \mathrm{~cm}^{3}\right)$; fluid in total composed $11.6 \%$ of the cerebellum. The vermis and fourth ventricle were no longer displaced from midline, reflecting a reduction in mass effect secondary to reduction in lesion size. However, the hydrocephalus was more prominent in the lateral recess of the fourth ventricle, as compared to the initial MRI.

The animal was severely impaired on acquisition of alternation in the T-maze, requiring 696 trials to reach criterion, defined as $85 \%$ or more correct trials on 2 consecutive 20-trial sessions. This finding was more than 2.32 standard deviations (139.4) above the mean trials to criterion (372.0) of 10 other California sea lions undergoing the same testing (Fig. 3). The next highest number of trials for any subject to reach criterion in this task was 511. Five of these 10 sea lions had healthy brains, while 5 had brain damage particular to the medial temporal lobe, suggesting that the impairment of the California sea lion in this study cannot be dismissed as a mere byproduct of general brain damage.

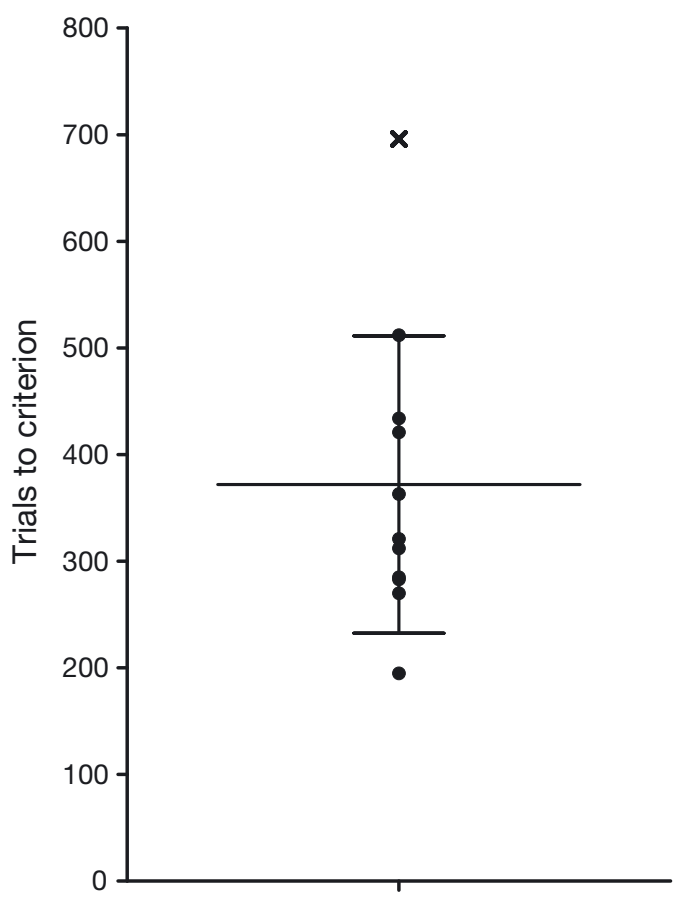

Fig. 3. Zalophus californianus. Number of trials to criterion, defined as $85 \%$ or more correct trials on 2 consecutive 20 -trial sessions in the T-maze. Mean $\pm \mathrm{SD}$ are represented. Each dot represents a sea lion. The California sea lion in this study is represented by an $x$
The animal was able to learn the location of the baited bucket in the once daily long-term spatial memory test. The animal went directly to the baited location without first visiting any of the non-baited buckets on the fifth and all 7 remaining days of testing. With 4 possible locations, the probability of correctly selecting the baited bucket on 7 consecutive days is astronomically small $(<0.0001)$. The other 10 animals also took part in the long-term spatial memory test. None of them reliably visited the correct bucket first prior to the third presentation.

Impairment in the T-maze suggests impaired procedural or motor learning, while the ability to remember the correct location of the baited bucket in the longterm memory test indicates some sparing of allocentric or associative memory. This suggests that the sea lion cerebellum is essential in guiding search behaviour and related learning, but not necessary for long-term location-based recall. These findings directly mirror those gleaned from extensive testing with rodents (Petrosini et al. 1996, Molinari \& Leggio 2007).

Following release the animal travelled approximately $75 \mathrm{~km}$ north and $80 \mathrm{~km}$ south of the release location before coming ashore in San Francisco Bay (Fig. 4). A total of 8200 dives were recorded and $90 \%$ of the dives were $40 \mathrm{~m}$ or less. Maximum dive depth increased over time from $40 \mathrm{~m}$ to approximately $120 \mathrm{~m}$ with a maximum recorded depth of $156 \mathrm{~m}$ (Fig. 5). The animal lost a third of its body weight in $25 \mathrm{~d}$, which is our best indication that the sea lion was not foraging efficiently. The maximum dive depths imply that this was not a physiological constraint, but this young animal may not have been an experienced feeder or may not have been behaviourally competent during this time.

Necropsy and histopathology confirmed that the sea lion died from subacute bronchopneumonia and pleuritis due to Pasteurella multocida infection. The status of nutrition score was 1/5, indicating emaciation. Grossly, there was mild fecal staining around the perineum, and the subcutaneous adipose was atrophic and mildly jaundiced. The thoracic cavity contained approximately $100 \mathrm{ml}$ of pale yellow viscous exudate. Cultures of this exudate grew large numbers of $P$. multocida. The lungs were diffusely congested and tracheobronchial lymph nodes enlarged. A bony callus at the mid-shaft of the left third, forth, and fifth ribs corresponded to the fractures noted on ante mortem radiographs.

Multifocal cavitations, up to $1.5 \times 1.0 \mathrm{~cm}$ were noted within the cerebellum. These cavitations contained a small amount of colorless fluid and were larger and more numerous on the right side of the cerebellum (Fig. 6). The cavitations were localized to white matter at the base of cerebellar folia and in some areas ex- 


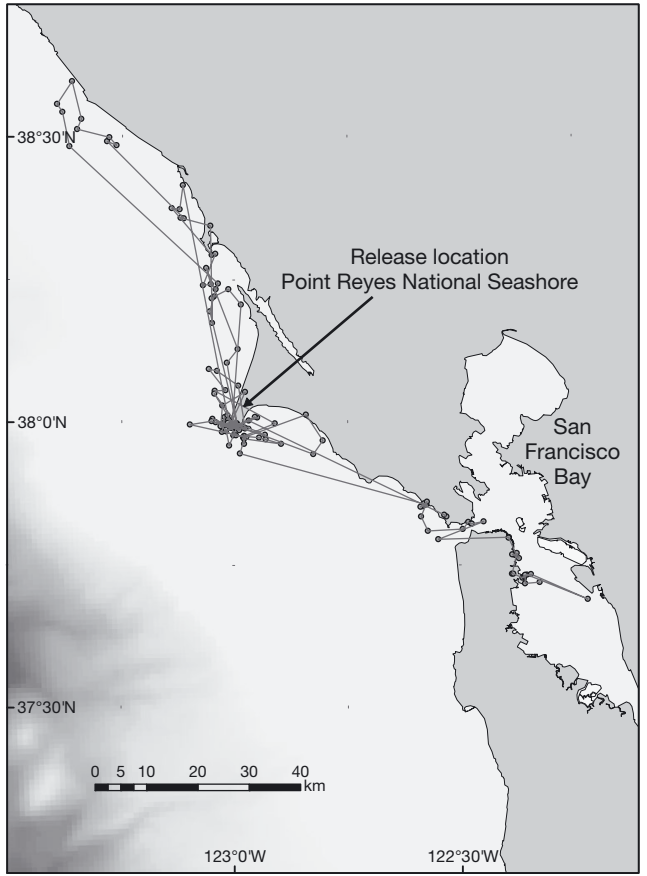

Fig. 4. Zalophus californianus. Satellite track data following release until re-stranding

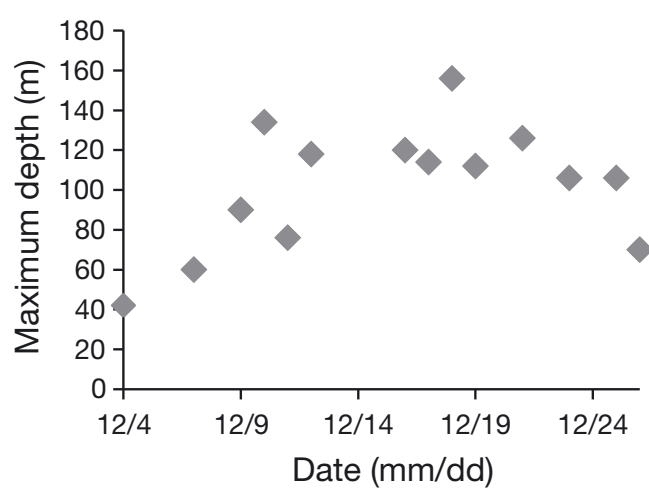

Fig. 5. Zalophus californianus. Maximum dive depths by date following release

tended outward towards the meninges. Histologically, the empty cerebellar cavitations were not lined by epithelium or ependyma and were surrounded by severely compressed and degenerated white matter that exhibited marked loss of axons and reactive gliosis. The adjacent cerebellar cortex was narrowed with loss of cells in the granular, molecular, and Purkinje cell layers. On sections stained with antibodies to Factor VIII there was no evidence of blood vessels associated with the cavitations. Several, small thick-walled, collapsed, hyalinized arteries were noted adjacent to cavitations. Within sections of lung, bronchioles and alveolar spaces contained numerous neutrophils, macrophages and necro-

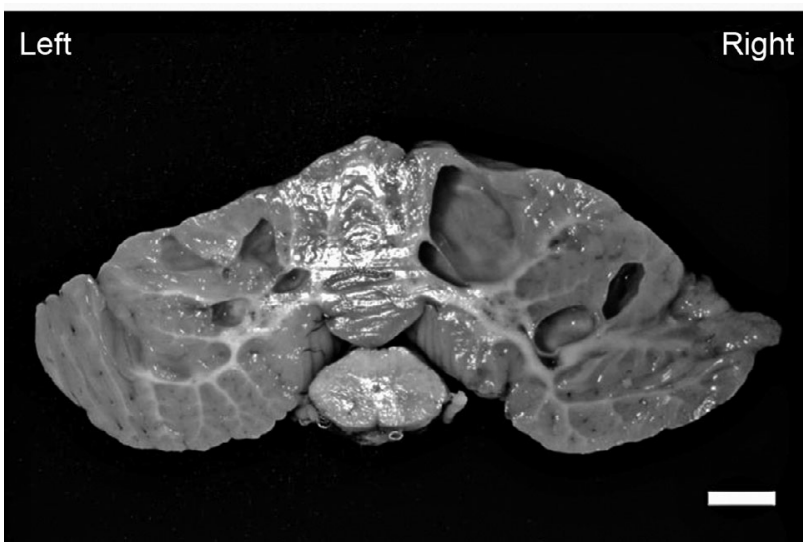

Fig. 6. Zalophus californianus. Transverse section of cerebellum with lesions. Scale bar $=1.0 \mathrm{~cm}$

tic debris with scattered Gram-negative coccobacilli. Bacterial morphology was consistent with the culture of Pasteurella multocida from pleural fluid. Pleural surfaces were edematous, contained similar inflammatory cells, and were lined by plump, reactive mesothelial cells. There was no evidence of pleural or parenchymal fibrosis, consistent with bronchopneumonia and pleuritis of subacute duration.

In bottlenose dolphins, adaptive modification of the intracranial circulation has been investigated and described (Ridgway et al. 2006). Although the neuroanatomy of the brain of the California sea lion was recently published, the vascular supply and perfusion of the brain has not been described (Montie et al. 2009). In the present study, it was not possible to determine whether gas emboli entered the pulmonary or somatic vasculature at the site of injury or arose in situ within the parenchyma of the cerebellum.

Although the pathogenesis of lesions observed in this California sea lion is most easily explained by the introduction of gas emboli at depth from the respiratory tract into the pulmonary intravascular compartment as a result of the rib fractures, this case is perplexing. Assuming gas did enter the pulmonary vasculature, emboli may have been small enough to pass through capillary beds at depth. If they circulated as a bolus but were located in the cerebellar vascular supply at ascent, gas may have entered the cerebellar parenchyma, expanded, and presented as we discovered them regardless of the original entry point. However, given that on histology there was no evidence of previous ischemic injury or hemorrhage, it is unlikely that the gas emboli lodged within and completely occluded cerebellar blood vessels. The resultant brain lesions instead are consistent with compression related damage from the space occupying gas and fluid accumulation.

Intraparenchymal accumulation of gas has also been noted in people with decompression sickness (Palmer 
et al. 1992, Oehmichen et al. 2006) and following cardiopulmonary resuscitation of individuals with pulmonary pathology (Cipriani et al. 2009). A retrospective search of over 15800 individual medical records at TMMC dating back to 1975 did not reveal any similar lesions in post mortem examinations or any similar clinical findings in successfully released animals in over 10 species of marine mammals. It is noteworthy, however, that many cases do not undergo the extensive diagnostics conducted in this instance, either ante or post mortem, and cases may have gone undiscovered.

We believe that the bronchopneumonia and pleuritis are not directly related to the rib fractures. The subacute inflammation more than $100 \mathrm{~d}$ from the initial finding of the bubbles and rib fractures, along with a lack of clinical evidence during the rehabilitation period indicates this problem developed following release. These are common sequelae to emaciation in this age class of sea lion brought to TMMC.

In summary, this case illustrates a rare, observed occurrence where the exquisite adaptations of a diving mammal to protect it from barotrauma have been disturbed resulting in gas embolism and accumulation in the brain. Although the animal apparently accommodated to the injury, remained clinically normal for several months until release, and dove to over $150 \mathrm{~m}$ depth, the altered cognitive function and marked weight loss noted on its return to TMMC suggested deficits in effective foraging behaviour. These findings suggest that morbidity and mortality in cases of barotrauma in marine mammals may be multifactorial. The true incidence and significance of this condition is unknown. Advanced diagnostic imaging in these animals is resource intensive but should be pursued to aid a more accurate determination of the incidence rate of barotrauma in marine mammals and underlying pathophysiology.

Acknowledgements. This work was conducted under a stranding agreement between NOAA National Marine Fisheries Service and TMMC. J. Adams, B. Bradley, and M. Boor of Cardiology Associates of Marin graciously provided imaging support. We thank Drs. M. Kinsel, D. Guzman, M. Moore and anonymous reviewers whose reviews significantly improved the quality and readability of the manuscript, along with K. Harle and A. Van Bonn for formatting assistance.

\section{LITERATURE CITED}

Cipriani NA, Hong C, Rosenblum J, Pytel P (2009) Air embolism with pneumocephalus. Arch Neurol 66: 1172-1173

Editorial responsibility: Michael Moore,

Woods Hole, Massachusetts, USA
Cox TM, Ragen TJ, Read AJ, Vos E and others (2006) Understanding the impact of anthropogenic sound on beaked whales. J Cetacean Res Manag 7:177-187

Elsner R (1999) Living in water: solutions to physiological problems. In: Reynolds JE, Rommel SA (eds) Biology of marine mammals. Smithsonian Institution Press, Washington, DC, p73-116

Falke KJ, Hill RD, Qvist J, Schneider RC and others (1985) Seal lungs collapse during free diving: evidence from arterial nitrogen tensions. Science 229:556-558

> Fernandez A, Edwards JF, Rodriguez F, Esopnosia de los Monteros A and others (2005) 'Gas and fat embolic syndrome' involving a mass stranding of beaked whales (family Ziphiidae) exposed to anthropogenic sonar signals. Vet Pathol 42:446-457

Gulland FMD, Hall AJ (2007) Is marine mammal health deteriorating? Trends in global reporting of marine mammal disease. EcoHealth 4:135-150

Houser DS, Dankiewicz-Talmadge L, Stockard TK, Ponganis PJ (2010) Investigation of the potential for vascular bubble formation in a repetitively diving dolphin. J Exp Biol 213:52-62

Jepson PD, Arbelo M, Deaville R, Patterson IAP and others (2003) Gas-bubble lesions in stranded cetaceans. Nature 425:575-576

Jepson PD, Deaville R, Paterson IAP, Pocknell AM and others (2005) Acute and chronic gas bubble lesions in cetaceans stranded in the United Kingdom. Vet Pathol 42:291-305

Molinari M, Leggio M (2007) Cerebellar information processing and visuospatial functions. Cerebellum 6:214-220

> Montie EW, Pussini N, Schnieder GE, Battey TWK and others (2009) Neuroanatomy and volumes of brain structures of a live California sea lion (Zalophus californianus) from magnetic resonance images. Anat Rec 292: $1523-1547$

> Moore MJ, Bogomolni AL, Dennison SE, Early G and others (2009) Gas bubbles in seals, dolphins, and porpoises entangled and drowned at depth in gillnets. Vet Pathol 46:536-547

Morris R (1984) Developments of a water-maze procedure for studying spatial learning in the rat. J Neurosci Methods 11:47-60

Oehmichen M, Auer M, König HG (2006) Forensic neuropathology and neurology. Springer, Berlin

> Palmer AC, Calder IM, Yates PO (1992) Cerebral vasculopathy in divers. Neuropathol Appl Neurobiol 18:113-124

> Petrosini L, Molinari M, DellAnna ME (1996) Cerebellar contribution to spatial event processing: Morris water maze and T-maze. Eur J Neurosci 8:1882-1896

Ridgway SH (1972) Homeostasis in the aquatic environment. In: Ridgway SH (ed) Mammals of the sea, biology and medicine. Charles C. Thomas, Springfield, IL

Ridgway SH, Howard R (1979) Dolphin lung collapse and intramuscular circulation during free diving: evidence from nitrogen washout. Science 206:1182-1183

Ridgway S, Houser D, Finneran J, Carder D and others (2006) Functional imaging of dolphin brain metabolism and blood flow. J Exp Biol 209:2902-2910

> Wood ER, Dudchenko PA, Robitsek RJ, Eichenbaum H (2000) Hippocampal neurons encode information about different types of memory episodes occurring in the same location. Neuron 27:623-633

Submitted: September 23, 2010; Accepted: May 24, 2011 Proofs received from author(s): August 20, 2011 